\title{
ON GEOMETRIC PARAMETRIZATION OF TEICHMÜLLER SPACES
}

\author{
MIKA SEPPÄLÄ and TUOMAS SORVALI
}

\section{Introduction}

We study geometric parametrizations of the Teichmüller space of an orientable or non-orientable $C^{\infty}$-surface which may have a non-empty boundary. Suppose that the dimension of the Teichmüller space is $n$. The problem is to find $n$ geodesics on the surface whose lengths parametrize the Teichmüller space in question. This problem can be solved explicitly if the surface is elementary or its fundamental group is free (cf. e.g. [TS 2] and [TS 3]). For a compact surface the problem turns out to be more complicated. It is well known that for oriented surfaces this problem can be solved locally. In this paper we present an explicit construction which gives a local parametrization for the Teichmüller space even in the case of non-orientable surfaces. Our basic reference in the theory of Teichmüller spaces is the forthcoming monograph [L] of O. Lehto.

\section{Parametrizations of two elementary Teichmüller spaces}

1. A hyperbolic Möbius transformation $g$ is uniquely determined by its attracting fixed point $a(g)$, its repelling fixed point $r(g)$ and its multiplier $k(g)$. For any point $z$ not fixed by $g$ it then holds

$$
k(g)=(g(z), z, r(g), a(g))=\frac{g(z)-r(g)}{g(z)-a(g)} \frac{z-a(g)}{z-r(g)} .
$$

Consider the set $\mathscr{P}$ whose elements are pairs $\left(g_{1}, g_{2}\right)$ of hyperbolic transformations with the following properties (Figure 1):

(i) $g_{1}$ and $g_{2}$ map the unit disk $D$ onto itself.

(ii) $r\left(g_{2}\right)=-1, a\left(g_{2}\right)=1$.

(iii) $y=\operatorname{Re}\left(r\left(g_{1}\right)\right)>0, \operatorname{Im}\left(r\left(g_{1}\right)\right)>0$.

(iv) $a\left(g_{1}\right)=-\overline{r\left(g_{1}\right)}$.

Supported by the Emil Aaltonen Foundation. 


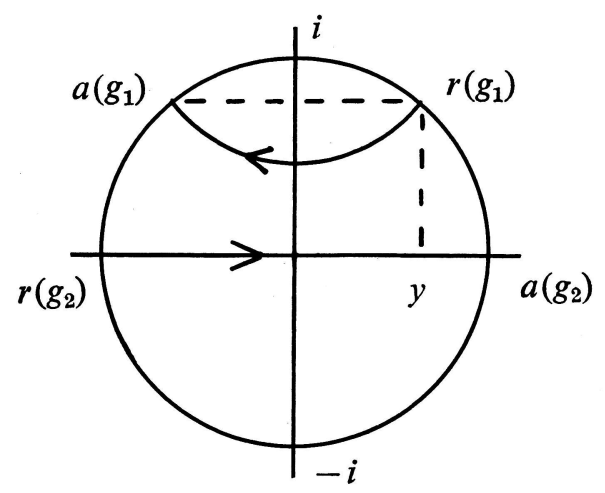

Figure 1

The real numbers

$$
\begin{aligned}
k_{1} & =k\left(g_{1}\right), \\
k_{2} & =k\left(g_{2}\right), \\
x & =\left(r\left(g_{1}\right), a\left(g_{1}\right), r\left(g_{2}\right), a\left(g_{2}\right)\right)
\end{aligned}
$$

determine $\left(g_{1}, g_{2}\right)$ uniquely and

$$
\left(g_{1}, g_{2}\right) \mapsto\left(k_{1}, k_{2}, x\right)
$$

defines a one-to-one mapping from $\mathscr{P}$ onto $I^{3}, I=\{t \in \boldsymbol{R} \mid t>1\}$.

Consider the function $f$,

$$
f(k)=\sqrt{k}+1 / \sqrt{k}
$$

If $k$ increases from 1 to $\infty$, then $f(k)$ increases from 2 to $\infty$.

Denote $k_{3}=k\left(g_{1} \circ g_{2}\right)$. By formula (4.11) in [TS 1],

$$
f\left(k_{3}\right)=\left|\frac{1}{1-x} f\left(k_{1} k_{2}\right)-\frac{x}{1-x} f\left(k_{1} / k_{2}\right)\right| .
$$

Inserting $x=\left(r\left(g_{1}\right),-\overline{r\left(g_{1}\right)},-1,1\right)=(1+y) /(1-y)$ and supposing

we get

$$
0<y<y_{1}=\frac{\left(k_{1}-1\right)\left(k_{2}-1\right)}{\left(k_{1}+1\right)\left(k_{2}+1\right)}
$$

$$
f\left(k_{3}\right)=\frac{1}{2}\left[\frac{f\left(k_{1} k_{2}\right)-f\left(k_{1} / k_{2}\right)}{y}-f\left(k_{1} k_{2}\right)-f\left(k_{1} / k_{2}\right)\right] .
$$

We keep $k_{1}$ and $k_{2}$ fixed and let $y$ vary between 0 and $y_{1}$. If $y$ is sufficiently small, then the isometric circles $I\left(g_{1}\right)$ and $I\left(g_{2}^{-1}\right)$ do not intersect (Figure 2).

The group $G$ generated by $g_{1}$ and $g_{2}$ is now discontinuous, $g_{1} \circ g_{2}$ is hyperbolic and the Riemann surface $D / G$ is conformally equivalent to a sphere from which 
three disks are removed, i.e., a pair of pants. The limiting case occurs when $I\left(g_{1}\right)$ and $I\left(g_{2}^{-1}\right)$ are tangent to each other. In this case $g_{1} \circ g_{2}$ is parabolic or $f\left(k_{3}\right)=2$ or

$$
y=y_{0}=\frac{\left(k_{1}-1\right)\left(k_{2}-1\right)}{\left(k_{1}+1\right)\left(k_{2}+1\right)+4 \sqrt{k_{1} k_{2}}} .
$$

We restrict the set $\mathscr{P}$ by requiring that also the following condition is satisfied:

$$
y<y_{0}=\frac{\left(k_{1}-1\right)\left(k_{2}-1\right)}{\left(k_{1}+1\right)\left(k_{2}+1\right)+4 \sqrt{k_{1} k_{2}}} .
$$

If $y$ increases from 0 to $y_{0}$, then $f\left(k_{3}\right)$ decreases from $\infty$ to 2 and $k_{3}$ decreases from $\infty$ to 1 . Hence $k_{1}, k_{2}$ and $k_{3}$ determine the pair $\left(g_{1}, g_{2}\right)$ uniquely and

$$
\left(g_{1}, g_{2},\right) \mapsto\left(k_{1}, k_{2}, k_{3}\right)
$$

defines a one-to-one mapping from $\mathscr{P}$ onto $I^{3}$.

2. Let $k$ be the set of pairs $\left(g_{1}, g_{2}\right)$ of hyperbolic transformations satisfying the following properties (Figure 3):

(i) $g_{1}$ and $g_{2}$ map the unit disk $D$ onto itself.

(ii) $r\left(g_{2}\right)=-1, a\left(g_{2}\right)=1$.

(iii) $a\left(g_{1}\right)=e^{i \varphi}, r\left(g_{1}\right)=-e^{i \varphi}, 0<\varphi<\pi$.

Denote $x=\left(r\left(g_{1}\right), a\left(g_{1}\right), r\left(g_{2}\right), a\left(g_{2}\right)\right), k_{1}=k\left(g_{1}\right), k_{2}=k\left(g_{2}\right)$ and $k_{3}=k\left(g_{1} \circ g_{2}\right)$. Then we have three parametrizations of $k$ :

$$
\begin{aligned}
& \left(g_{1}, g_{2}\right) \mapsto\left(k_{1}, k_{2}, \varphi\right), \\
& \left(g_{1}, g_{2}\right) \mapsto\left(k_{1}, k_{2}, x\right), \\
& \left(g_{1}, g_{2}\right) \mapsto\left(k_{1}, k_{2}, k_{3}\right),
\end{aligned}
$$

where $1<k_{j}<\infty, j=1,2,3,-\infty<x<0$ and $0<\varphi<\pi$. We consider the interdependence of $\varphi, x$, and $k_{3}$.

A direct calculation yields

$$
x=-(\tan \varphi / 2)^{2}
$$

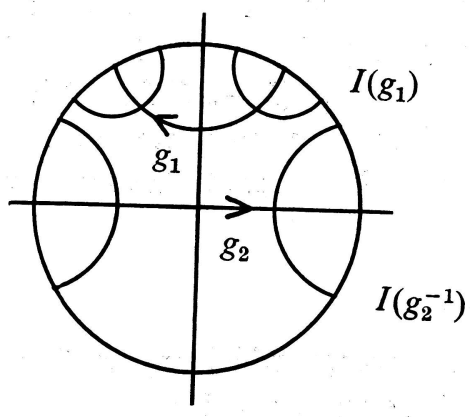

Figure 2

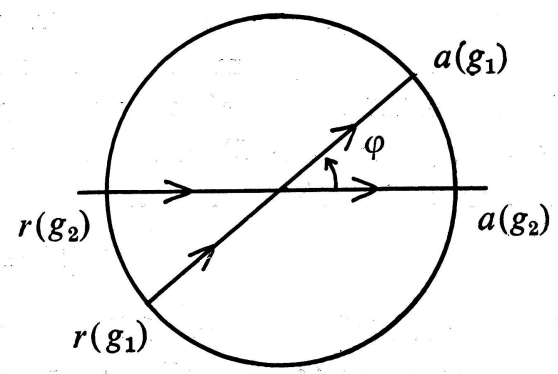

Figure 3 
Applying formula (4.11) in [TS 1] we have now

$$
f\left(k_{3}\right)=\frac{1}{1-x} f\left(k_{1} k_{2}\right)-\frac{x}{1-x} f\left(k_{1} / k_{2}\right) .
$$

Hence $f\left(k_{3}\right)$ increases from $f\left(k_{1} / k_{2}\right)$ to $f\left(k_{1} k_{2}\right)$ as $x$ increases from $-\infty$ to 0 .

Inserting $x=-(\tan \varphi / 2)^{2}$ we get

$$
\begin{aligned}
& f\left(k_{3}\right)=\frac{1}{2}\left[(1+\cos \varphi) f\left(k_{1} k_{2}\right)+(1-\cos \varphi) f\left(k_{1} / k_{2}\right)\right] \\
& =\frac{1}{2}\left[\cos \varphi\left(f\left(k_{1} k_{2}\right)-f\left(k_{1} / k_{2}\right)\right)+f\left(k_{1} k_{2}\right)+f\left(k_{1} / k_{2}\right)\right] .
\end{aligned}
$$

The set $\mathscr{P}$ is actually a model for the Teichmüller space of a pair of pants. Similarly, the set $k$ represents the Teichmüller space of a torus from which a disk is removed. The method we used to parametrize them admits a generalization to all freely generated Fuchsian groups (cf. e.g. [TS 2] and [TS 3]). In a forthcoming paper [SS] we apply the above technique to the Teichmüller spaces of all compact surfaces of genus at least two.

Also topological methods can be applied here if the orbit space of the corresponding Riemann surface is compact. In order to describe how this can be done it is necessary to review a topological approach to Teichmüller spaces.

\section{Teichmüller spaces of hyperbolic metrics}

3. Compact surfaces whose Euler-Poincaré characteristic is non-negative are elementary. We suppose from now on that $\Sigma$ is a compact and not elementary $C^{\infty}$-surface which may have a non-empty boundary. This excludes the following surfaces: the sphere, the real projective plane, the disk, the annulus, the Möbius band, the torus, the complex plane and the Klein bottle. These surfaces can be treated by explicit methods.

All surfaces that are not elementary carry hyperbolic metrics. These metrics correspond to conformal structures of the surfaces and can be recovered from the hyperbolic metric of the unit disk via the uniformization theorem. Let $\mathscr{M}(\Sigma)$ be the set of complete hyperbolic metrics on the non-elementary $C^{\infty}$-surface $\Sigma$.

A diffeomorphism $h: \Sigma \rightarrow \Sigma$ induces a mapping $h^{*}: \mathscr{M}(\Sigma) \rightarrow \mathscr{M}(\Sigma) ; h^{*}(m)$ for an $m \in \mathscr{M}(\Sigma)$ is defined requiring $h:\left(\Sigma, h^{*}(m)\right) \rightarrow(\Sigma, m)$ be isometric. In this way the group $\operatorname{Diff}_{0}(\Sigma)$ of diffeomorphic self-mappings of $\Sigma$ isotopic to the identity acts on $\mathscr{M}(\Sigma)$. The orbit space

$$
T(\Sigma)=\mathscr{M}(\Sigma) / \operatorname{Diff}_{0}(\Sigma)
$$

is the Teichmüller space of $\Sigma$. It carries a standard topology which makes $T(\Sigma)$ homeomorphic to a Euclidean space. The surface $\Sigma$ being oriented and without boundary, $T(\Sigma)$ is also a complex manifold. For non-orientable surfaces or surfaces 
with boundary $T(\Sigma)$ is a real analytic manifold. We assume the standard results about Teichmüller spaces known. The forthcoming monograph of Lehto [L] is our basic reference.

If $\Sigma$ is not orientable we may form its oriented double cover $\pi: \Sigma^{d} \rightarrow \Sigma$ whose covering group is generated by an orientation reversing involution $\sigma: \Sigma^{d} \rightarrow \Sigma^{d}$ which does not have fixed-points. If $\langle\sigma\rangle$ is the group generated by $\sigma, \quad \Sigma=\Sigma^{d} /\langle\sigma\rangle$.

The projection $\pi: \Sigma^{d} \rightarrow \Sigma$ defines $\pi^{*}: \mathscr{M}(\Sigma) \rightarrow \mathscr{M}\left(\Sigma^{d}\right)$ where $\pi^{*}(m)$ for an $m \in \mathscr{M}(\Sigma)$ is defined by requiring $\pi:\left(\Sigma^{d}, \pi^{*}(m)\right) \rightarrow(\Sigma, m)$ be isometric. This mapping $\pi^{*}$ induces an injective mapping $\pi^{*}: T(\Sigma) \rightarrow T\left(\Sigma^{d}\right)$ for which $\pi^{*}(T(\Sigma))=T\left(\Sigma^{d}\right)_{\sigma^{*}}$. Here $T\left(\Sigma^{d}\right)_{\sigma^{*}}$ is the set of fixed-points of the induced mapping $\sigma^{*}: T\left(\Sigma^{d}\right) \rightarrow T\left(\Sigma^{d}\right)$ (cf. [MS]).

4. A disk with two holes is a pair of pants. It is a building block for compact oriented surfaces. In order to study Teichmüller spaces we should first parametrize hyperbolic metrics of pairs of pants. This is, of course, well known. We will, however, briefly indicate how the considerations of Section 1 can be applied here.

Let $P$ be a fixed topological pair of pants. Denote the boundary components of $P$ by $\gamma_{1}, \gamma_{2}$ and $\gamma_{3}$. A complete hyperbolic metric $m$ on $P$ corresponds to a complex structure $X$ of $P .(P, X)$ is a Riemann surface whose universal covering is the unit disk which can be normalized in such a way that the covering group $G$ is freely generated by Möbius transformations $g_{1}$ and $g_{2}$ in $\mathscr{P}$. The hyperbolic metric of the unit disks then gives the metric $m$ of $(P, X)$.

Denote the hyperbolic length of the geodesic curve homotopic to $\gamma_{j}$ by $l_{m}\left\{\gamma_{j}\right\}$. It is immediate that

$$
L: T(P) \rightarrow\left(\boldsymbol{R}_{+}^{*}\right)^{3},[m] \mapsto\left(l_{m}\left\{\gamma_{1}\right\}, l_{m}\left\{\gamma_{2}\right\}, l_{m}\left\{\gamma_{3}\right\}\right)
$$

is a well-defined mapping. Here $\boldsymbol{R}_{+}^{*}=\{r \in \boldsymbol{R} \mid r>0\}$.

\section{Proposition 4.1. The mapping $L: T(P) \rightarrow\left(\boldsymbol{R}_{+}^{*}\right)^{3}$ is a homeomorphism.}

Proof. Let us first show that $L$ is one-to-one. Consider points $[m]$ and $\left[m^{\prime}\right]$ in $T(P)$ with $L([m])=L\left(\left[m^{\prime}\right]\right)$. The hyperbolic metrics $m$ and $m^{\prime}$ can be recovered from the hyperbolic metric of the unit disk by the identifications $(P, m)=D / G$ and $\left(P, m^{\prime}\right)=D / G^{\prime}$ where $G$ is freely generated by $g_{1}$ and $g_{2}$ in $\mathscr{P}$ and $G^{\prime}$ is likewise freely generated by $g_{1}^{\prime}$ and $g_{2}^{\prime}$ in $\mathscr{P}$. Write $g_{3}=g_{2} \circ g_{1}, g_{3}^{\prime}=g_{2}^{\prime} \circ g_{1}^{\prime}$ and assume that $g_{j}$ and $g_{j}^{\prime}$ both cover $\gamma_{j}, j=1,2,3$.

Let $k_{j}$ be the multiplier of $g_{j}$ and $k_{j}^{\prime}$ that of $g_{j}^{\prime}, j=1,2,3$. Now $l_{m}\left\{\gamma_{j}\right\}=\log k_{j}$ and $l_{m^{\prime}}\left\{\gamma_{j}\right\}=\log k_{j}^{\prime}$. Hence $k_{j}=k_{j}^{\prime}$ for $j=1,2,3$. Consequently, $g_{1}=g_{1}^{\prime}$ and $g_{2}=g_{2}^{\prime}$, and $G=G^{\prime}$.

Now the identity mapping of $D$ induces an isometry $(P, m) \rightarrow\left(P, m^{\prime}\right)$ which maps $\gamma_{j}$ onto itself for $j=1,2,3$. Since it is also sense preserving it must be homotopic to the identity mapping of $P$. This means that $m$ and $m^{\prime}$ define the same point in $T(P)$, and the mapping $L$ is injective. 
The mapping $L$ is also onto since $\left(g_{1}, g_{2}\right) \mapsto\left(k_{1}, k_{2}, k_{3}\right)$ defines a surjection of $\mathscr{P}$ onto $I^{3}$. This shows that $L$ is bijective.

To complete the proof it suffices to show that $L$ is continuous. But this is an immediate consequence of Theorem 4.1 in [TS 1].

\section{Construction of the local parametrization}

5. Let $\Sigma$ be a compact and not elementary oriented $C^{\infty}$-surface without boundary. It can be decomposed into $-\chi(\Sigma)=2 g-2$ pairs of pants. Here $g$ is the genus of $\Sigma$. Fix a decomposition $\left(P_{1}, P_{2}, \ldots, P_{2 g-2}\right)$ of $\Sigma$ into pairs of pants $P_{j}$. We assume that each $P_{j}$ is the image of a standard pair of pants $P$ under an embedding. Let $\alpha_{1}, \alpha_{2}, \ldots, \alpha_{3 g-3}$ be the boundary curves of the pairs of pants in the above decomposition. Each $\alpha_{j}$ is a simple closed curve on $\Sigma$ and appears as a boundary component of two different pairs of pants.

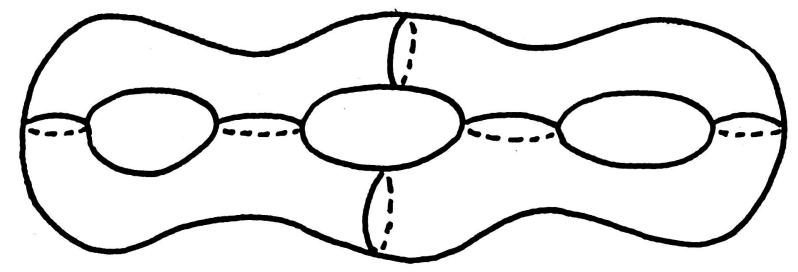

Figure 4. A decomposition of a genus 3 surface into pairs of pants

Each point of the Teichmüller space $T(\Sigma)$ of $\Sigma$ can be represented by a complete hyperbolic metric $m$ of $\Sigma$ for which each $\alpha_{j}, j=1,2, \ldots, 3 g-3$, is a geodesic. Hence there is no loss in generality in assuming that all the hyperbolic metrics under consideration have this property.

Like in the case of pairs of pants

$$
L: T(\Sigma) \rightarrow\left(\boldsymbol{R}_{+}^{*}\right)^{3 g-3}, \quad[m] \mapsto\left(l_{m}\left\{\alpha_{1}\right\}, \ldots, l_{m}\left\{\alpha_{3 g-3}\right\}\right)
$$

is a well-defined mapping.

It follows, from Proposition 4.1, that the mapping (5.1) is onto. It is also real analytic with respect to the complex structure of $T(\Sigma)$ but we do not need this fact.

Following A. Douady ([TT], Exposé 7) we study the mapping (5.1) introducing an action of $R^{3 g-3}$ on $T(\Sigma)$.

Orient the curves $\alpha_{j}$ and choose collars $\alpha_{j} \times[0,1]$ on the left side of $\alpha_{j}=\alpha_{j} \times\{0\}$. Assume that these collars are disjoint. For a complete hyperbolic metric $m$ of $\Sigma$ and each number $t \in \boldsymbol{R}$ define a diffeomorphism $\varphi_{j}(m, t)$ of $\alpha_{j} \times[0,1]$ onto itself with the following properties: 
1) $\varphi_{j}(m, t)$ is the identity in a neighbourhood of $\alpha_{j} \times\{1\}$.

2) $\varphi_{j}(m, t)$ is an isometry of $m$ in a neighbourhood of $\alpha_{j} \times\{0\}$.

3) A lifting of $\varphi_{j}(m, t)$ to the universal cover $\boldsymbol{R} \times[0,1]$ of $\alpha_{j} \times[0,1]$ is the identity on $\boldsymbol{R} \times\{1\}$ and a translation of length $t l_{m}\left\{\alpha_{j}\right\}$ on $\boldsymbol{R} \times\{0\}$ which covers $\alpha_{j} \times\{0\}$.

Here we consider $\boldsymbol{R} \times[0,1]$ with a lifting of the metric $m$.

The deformed metric $\theta_{j}(m, t)$ is now defined by $\theta_{j}(m, t)=\varphi_{j}^{*}(m, t)(m)$ on $\alpha_{j} \times[0,1]$ and by $\theta_{j}(m, t)=m$ elsewhere.

For $\left(t_{1}, t_{2}, \ldots, t_{3 g-3}\right) \in \boldsymbol{R}^{3 g-3}, \theta\left(m, t_{1}, t_{2}, \ldots, t_{3 g-3}\right)$ is the metric defined by $\theta_{j}\left(m, t_{j}\right)$ in $\alpha_{j} \times[0,1], j=1,2, \ldots, 3 g-3$, and by $m$ elsewhere.

Note that the above construction (cf. e.g. [TT], page 129) gives a hyperbolic metric since all the curves $\alpha_{j}$ were assumed to be geodesics of the metric $m$.

We now quote a basic result ([TT], Exposé 7, Proposition 3, p. 133).

Proposition 5.1. The mapping (5.1) $L: T(\Sigma) \rightarrow\left(\boldsymbol{R}_{+}^{*}\right)^{3 g-3}$ is a principal $\boldsymbol{R}^{3 g-3}$ fibration.

We continue to follow the construction of [TT], Exposé 7. For each index $j$ consider the pairs of pants $P_{j_{1}}$ and $P_{j_{2}}$ adjacent to $\alpha_{j}$. Let $\beta_{j}$ be a simple closed curve in $P_{j_{1}} \cup P_{j_{2}}$ which is neither homotopic to any of the boundary components of $P_{j_{1}}$ or $P_{j_{2}}$ nor homotopic to a point. Fix the curves $\beta_{1}, \beta_{2}, \ldots, \beta_{3 g-3}$.

For a hyperbolic metric $m$ we can now consider the function

$$
t \mapsto l_{\theta_{j}(m, t)}\left\{\beta_{j}\right\}
$$

where $l_{\theta_{j}(m, t)}\left\{\beta_{j}\right\}$ is the length of the geodesic curve homotopic to $\beta_{j}$ in the deformed metric $\theta_{j}(m, t)$.

Proposition 5.2. ([TT], Exposé 7, Proposition 2, p. 130.) The function (5.2) is strictly convex and has a unique minimum.

We will use the above results to find a local geometric parametrization for the Teichmüller space $T(\Sigma)$ in terms of lengths of $n$ simple closed curves where $n=$ $\operatorname{dim}_{R} T(\Sigma)$.

Proposition 5.1 means that the mapping (5.1)

$$
L: T(\Sigma) \rightarrow\left(\boldsymbol{R}_{+}^{*}\right)^{3 g-3}
$$

has a continuous global section $s:\left(\boldsymbol{R}_{+}^{*}\right)^{3 g-3} \rightarrow T(\Sigma)$, and that for each $[m] \in T(\Sigma)$ there is a unique $\left(t_{1}^{m}, \ldots, t_{3 g-3}^{m}\right) \in \boldsymbol{R}^{3 g-3}$ such that

$$
[m]=\left[\theta\left(s \circ L([m]), t_{1}^{m}, \ldots, t_{3 g-3}^{m}\right)\right] .
$$

Furthermore, the mapping

$$
\Psi_{s}: T(\Sigma) \rightarrow\left(\boldsymbol{R}_{+}^{*}\right)^{3 g-3} \times \boldsymbol{R}^{3 g-3}, \quad[m] \mapsto\left(L([m]), t_{1}^{m}, \ldots, t_{3 g-3}^{m}\right),
$$

is a homeomorphism of $T(\Sigma)$ which depends on the section $s$.

Let us now try to get a more detailed idea of the mapping (5.3). Fix a $j$, $1 \leqq j \leqq 3 g-3$. For a hyperbolic metric $m$, the length $l_{m}\left\{\beta_{j}\right\}$ of the geodesic curve 
homotopic to $\beta_{j}$ depends on the point $L([m]) \in\left(\boldsymbol{R}_{+}^{*}\right)^{3 g-3}$ and on $t_{j}^{m}$ but not on the coordinates $t_{k}^{m}, k \neq j$. This follows immediately from the definition of $t_{k}^{m}$.

Given an $m$ the function (5.2) has a unique minimum point $\left(t_{j}^{m}\right)_{0}$ by Proposition 5.2. If $s^{\prime}:\left(R_{+}^{*}\right)^{3 g-3} \rightarrow T(\Sigma)$ is a continuous section of the mapping (5.1) then for each $x \in\left(\boldsymbol{R}_{+}^{*}\right)^{3 g-3}$ we may consider the points $\left(t_{j}^{s^{\prime}(x)}\right)_{0}$. For each $j$ that point is the unique minimum point of a strictly convex function which depends continuously on the parameter $x \in\left(\boldsymbol{R}_{+}^{*}\right)^{3 g-3}$. Such a minimum point depends continuously on $x$ as well. It follows that

$$
s:\left(\boldsymbol{R}_{+}^{*}\right)^{3 g-3} \rightarrow T(\Sigma), \quad x \mapsto\left[\theta\left(s^{\prime}(x),\left(t_{1}^{s^{\prime}(x)}\right)_{0}, \ldots,\left(t_{3 g-3}^{s^{\prime}(x)}\right)_{0}\right)\right]
$$

is a continuous section of the mapping (5.1).

That section has the following property: For each $x \in\left(\boldsymbol{R}_{+}^{*}\right)^{3 g-3}$ all the functions

$$
t \mapsto l_{\theta_{j}(s(x), t)}\left\{\beta_{j}\right\}, \quad j=1,2, \ldots, 3 g-3,
$$

are strictly increasing for $t>0$ and strictly decreasing for $t<0$.

Consider the parametrization (5.3) of $T(\Sigma)$ associated with this section $s$. Let

$$
V=\left\{\left(x, t_{1}, \ldots, t_{3 g-3}\right) \in\left(\boldsymbol{R}_{+}^{*}\right)^{3 g-3} \times \boldsymbol{R}^{3 g-3} \mid x \in\left(\boldsymbol{R}_{+}^{* 3 g}\right)^{-3}, \quad t_{j} \neq 0 \text { for all } j\right\}
$$

and $V_{s}=\Psi_{s}^{-1}(V) . V_{s}$ is clearly an open set of $T(\Sigma)$ since $\Psi_{s}$ is continuous.

The following result is now almost obvious.

\section{Proposition 5.3. The mapping}

$$
\mathscr{L}: T(\Sigma) \rightarrow\left(\boldsymbol{R}_{+}^{*}\right)^{6 g-6}, \quad[m] \mapsto\left(l_{m}\left\{\alpha_{1}\right\}, l_{m}\left\{\beta_{1}\right\}, \ldots, l_{m}\left\{\alpha_{2 g-3}\right\}, l_{m}\left\{\beta_{3 g-3}\right\}\right)
$$

is a local homeomorphism on $V_{s}$.

Proof. It suffices to show that $\mathscr{L} \circ\left(\Psi_{s}\right)^{-1}$ is a local homeomorphism on $V$. This is, on the other hand, clear since for $t_{j} \neq 0$ each function

$$
t_{j} \mapsto l_{\theta_{j}\left(s(x), t_{j}\right)}\left\{\beta_{j}\right\}
$$

is a local homeomorphism. Hence the length of the curve $\beta_{j}$ locally parametrizes the coordinate $t_{j}$ in $V$. This proves the proposition.

To get a local parametrization at points of $T(\Sigma)$ which do not belong to $V_{s}$ we simply replace some (or all) of the curves $\beta_{j}$ by their Dehn twists around the corresponding $\alpha_{j}$. In that way we get a local parametrization for $T(\Sigma)$ everywhere.

\section{Parametrization of non-orientable surfaces}

6. Let $\Sigma$ be a compact non-orientable $C^{\infty}$-surface without boundary. Consider its orientable double cover $\pi: \Sigma^{d} \rightarrow \Sigma$ and assume that $\Sigma^{d}$ is of genus $g, g \geqq 2$. For our considerations it is necessary to get a concrete picture of the covering $\pi: \Sigma^{d} \rightarrow \Sigma$.

Non-orientable surfaces with homeomorphic double covers are homeomorphic. Hence it suffices to find one concrete non-orientable surface $\Sigma$ whose orientable 
double cover $\Sigma^{d}$ is of genus $g$. This amounts to finding an orientation reversing involution $\sigma: \Sigma^{d} \rightarrow \Sigma^{d}$, which does not have fixed points, of an oriented surface $\Sigma^{d}$ of genus $g$. Because if $\sigma$ is such an involution of $\Sigma^{d}$ then $\Sigma=\Sigma^{d} /\langle\sigma\rangle$ is a nonorientable surface without boundary whose orientable double cover is $\Sigma^{d}$.

To construct the above involution take first two copies of a sphere from which $g+1$ disks have been removed. Glue these spheres with holes together along the boundary. In that way we obtain an oriented surface $\Sigma^{d}$ of genus $g$. This surface is a ramified double cover of the original sphere with two holes. Let $\tau: \Sigma^{d} \rightarrow \Sigma^{d}$ be the sheet interchange of this double cover. Let $\alpha_{1}, \ldots, \alpha_{g+1}$ be the components of the fixed-point set of $\tau$; they are just simple closed curves on $\Sigma^{d}$ which correspond to the boundary curves of the sphere with holes we started with.

Let $A_{j}$ be a tubular neighbourhood of $\alpha_{j}, j=1,2, \ldots, g+1$. Assume that $A_{j} \cap A_{k}=\emptyset$ for $j \neq k$. We may further assume that $\tau\left(A_{j}\right)=A_{j}$ for $j=1,2, \ldots, g+1$.

We choose the universal coverings $\tilde{A}_{j} \rightarrow A_{j}$ of the annuli $A_{j}$ in such a way that $\tilde{A}_{j}=\{z \in \boldsymbol{C} \mid-1<\operatorname{Im} z<+1\}$, the real axis covers $\alpha_{j}$, the complex conjugation is a lifting of $\left.\tau\right|_{A_{j}}$, and the covering group of $\tilde{A}_{j} \rightarrow A_{j}$ is generated by the translation $z \mapsto z+2$.

The mapping $\tilde{f}_{j}: \tilde{A}_{j} \rightarrow \tilde{A}_{j}, \tilde{f}(z)=z+(1-\operatorname{Im} z)$, induces a homeomorphism $A_{j} \rightarrow A_{j}$ which is the identity on the boundary of $A_{j}$. Let $f: \Sigma^{d} \rightarrow \Sigma^{d}$ be the orientation preserving homeomorphism of $\Sigma^{d}$ which, in each $A_{j}$, agrees with the above mapping and is the identity in $\Sigma^{d}-\left(\bigcup_{j=1}^{g+1} A_{j}\right)$. The following lemma is an immediate consequence of all the definitions. The idea we have used here is due to B. Mazur and H. Jaffee.

Lemma. The mapping $\sigma=f \circ \tau$ is an orientation reversing involution of $\Sigma^{d}$ which does not have fixed points.

To get a better idea of $\sigma$ consider a simple closed curve $\beta_{j}$ which intersects $\alpha_{j}$ as in the picture below (Figure 5). Assume that $\tau\left(\beta_{j}\right)$ is homotopic to $\beta_{j}$. Then $\sigma\left(\beta_{j}\right)$ is homotopic to the Dehn twist of $\beta_{j}$ around $\alpha_{j}$ (to a suitable direction).
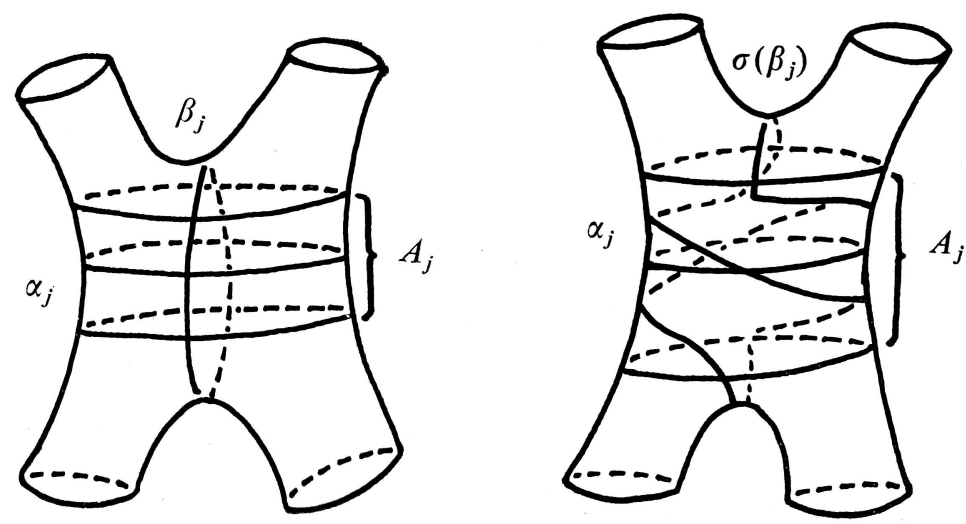

Figure 5. The curve $\sigma\left(\beta_{j}\right)$ is homotopic to the Dehn twist of $\beta_{j}$ 
This mapping $\sigma$ is explicit enough in order to study the Teichmüller space of the non-orientable surface $\Sigma=\Sigma^{d} /\langle\sigma\rangle$ of genus $g$.

Identify first $T(\Sigma)$ with $T\left(\Sigma^{d}\right)_{\sigma^{*}}$, the fixed-point set of $\sigma^{*}: T\left(\Sigma^{d}\right) \rightarrow T\left(\Sigma^{d}\right)$. We can do this by the considerations of Section 3.

Our aim is to find $3 g-3=\operatorname{dim}_{R} T(\Sigma)=(1 / 2) \operatorname{dim}_{R} T\left(\Sigma^{d}\right)$ simple closed curves on $\Sigma$ whose lengths parametrize $T(\Sigma)$ locally on an open set of $T(\Sigma)$.

By Proposition 5.3 the points $[m]$ of $T\left(\Sigma^{d}\right)$ can locally be parametrized by the lengihs of $6 g-6$ geodesic curves. We will now show that $\sigma^{*}([m])=[m]$ implies simple relations for the lengths of the parametrizing curves in such a way that only $3 g-3$ curves suffice to parametrize $T\left(\Sigma^{d}\right)_{\sigma^{*}}$ locally. The projections of these curves to $\Sigma^{d} /\langle\sigma\rangle=\Sigma$ are then simple closed curves on $\Sigma$ whose lengths parametrize $T(\Sigma)$.

Note first that we can decompose $\Sigma^{d}$ into pairs of pants is such a way that the following holds:

1) The components of the fixed-point set of the involution $\tau: \Sigma^{d} \rightarrow \Sigma^{d}, \alpha_{1}, \alpha_{2}, \ldots$, $\alpha_{g+1}$, are boundary curves of some pairs of pants.

2) Let $\alpha_{g+2}, \ldots, \alpha_{3 g-3}$ be the remaining boundary curves. The involution $\sigma$ of $\Sigma^{d}$ permutes these curves.

These conditions imply that $\sigma$ maps each $\alpha_{j}, j=1,2, \ldots, g+1$, onto itself and each $\alpha_{k}, k>g+1$, onto some other $\alpha_{k^{\prime}}$. Let us choose the indices in such a way that $\sigma\left(\alpha_{g+1+k}\right)=\alpha_{2 g-1+k}$ for $k=1,2, \ldots, g-2$.

We can further choose the curves $\beta_{j}$ associated with the boundary curves $\alpha_{j}$ in such a way that for $j=1,2, \ldots, g+1, \tau\left(\beta_{j}\right)=\beta_{j}$ and $\sigma\left(\beta_{g+1+k}\right)=\beta_{2 g-1+k}$ for $k=1,2, \ldots, g-2$. Then, for $j=1,2, \ldots, g+1, \sigma\left(\beta_{j}\right)$ is homotopic to the Dehn twist of $\beta_{j}$ around $\alpha_{j}$.

Let $s:\left(\boldsymbol{R}_{+}^{*}\right)^{3 g-3} \rightarrow T\left(\Sigma^{d}\right)$ be the section (5.4) of the fibration (5.1), and let $V_{s}$ be the open set of $T\left(\Sigma^{d}\right)$ of Proposition 5.3. Then $V_{s} \cap T\left(\Sigma^{d}\right)_{\sigma^{*}}$ is open in $T\left(\Sigma^{d}\right)_{\sigma^{*}}$.

Proposition 5.4. The lengths of the $3 g-3$ geodesic curves hornotopic to $\alpha_{1}, \ldots, \alpha_{2 g-1}$ and $\beta_{g+2}, \ldots, \beta_{2 g-1}$ parametrize $T\left(\Sigma^{d}\right)_{\sigma^{*}}$ locally on $V_{s} \cap T\left(\Sigma^{d}\right)_{\sigma^{*}}$.

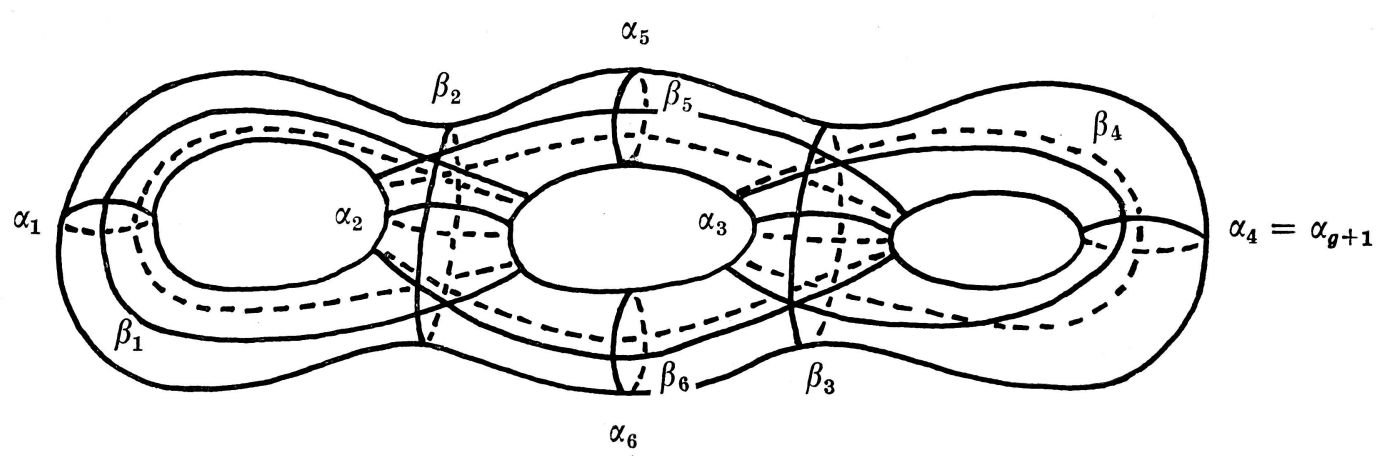

Figure 6. Here is a genus 3 surface with the curves $\alpha_{j}$ and $\beta_{j}$ 
Proof. Let $[m] \in T\left(\Sigma^{d}\right)_{\sigma^{*}}$. In view of Proposition 5.3 it suffices to show that the numbers $l_{m}\left\{\alpha_{j}\right\}$ and $l_{m}\left\{\beta_{j}\right\}, j=1,2, \ldots, 3 g-3$ are locally uniquely defined by $l_{m}\left\{\alpha_{j}\right\}, j=1,2, \ldots, 2 g-1$ and $l_{m}\left\{\beta_{j}\right\}, j=g+2, \ldots, 2 g-1$. To that end we use the fact that the involution $\sigma:\left(\Sigma^{d}, m\right) \rightarrow\left(\Sigma^{d}, m\right)$ is an isometry (the point $[m] \in T\left(\Sigma^{d}\right)$ is kept fixed by $\sigma^{*}$ ).

Consequently, since $\alpha_{2 g-1+k}=\sigma\left(\alpha_{g+1+k}\right)$ for $k=1,2, \ldots, g-2$, the lengths of $\alpha_{1}, \ldots, \alpha_{2 g-1}$ determine the lengths of all the curves $\alpha_{j}$. In the same way the lengths of the geodesic curves homotopic to $\beta_{2 g}, \ldots, \beta_{3 g-3}$ are determined by the numbers $l_{m}\left\{\beta_{g+2}\right\}, \ldots, l_{m}\left\{\beta_{2 g-1}\right\}$. It remains to show that the numbers $l_{m}\left\{\beta_{1}\right\}, \ldots, l_{m}\left\{\beta_{g+1}\right\}$ are uniquely determined.

Let $s$ be the above section of the fibration (5.1). Consider the parametrization (5.3) $\Psi_{s}: T\left(\Sigma^{d}\right) \rightarrow\left(\boldsymbol{R}_{+}^{*}\right)^{3 g-3} \times \boldsymbol{R}^{3 g-3}$ associated with this section. Note first that by definitions

$$
l_{\theta\left(s(x), t_{1}, \ldots, t_{3 g-3}\right)}\left\{\beta_{j}\right\}=l_{\theta_{j}\left(s(x), t_{j}\right)}\left\{\beta_{j}\right\}
$$

for all $j=1,2, \ldots, 3 g-3$ and $x \in\left(\boldsymbol{R}_{+}^{*}\right)^{3 g-3}$.

Assume that $\left(x, t_{1}, \ldots, t_{3 g-3}\right)=\Psi_{s}([m])$. Consider the function (5.2) $t \mapsto$ $l_{\theta_{j}(s(x), t)}\left\{\beta_{j}\right\}$. By the choice of the curves $\beta_{j}, \sigma\left(\beta_{j}\right)$ is homotopic to the Dehn twist of $\beta_{j}$ around $\alpha_{j}$. Hence, since $\sigma$ is an isometry of the metric $m$,

$$
l_{m}\left\{\sigma\left(\beta_{j}\right)\right\}=l_{\theta_{j}\left(s(x), t_{j}\right)}\left\{\sigma\left(\beta_{j}\right)\right\}=l_{\theta_{j}\left(s(x), t_{j}+1\right)}\left\{\beta_{j}\right\}=l_{\theta_{j}\left(s(x), t_{j}\right.}\left\{\beta_{j}\right\} .
$$

The function (5.2) is strictly convex with a unique minimum. Hence there is only one value of $t_{j}$ for which (6.2) can hold. This implies the proposition.

Proposition 5.4 gives a local parametrization for $T(\Sigma)$ in the open set corresponding to $V_{s} \cap T\left(\Sigma^{d}\right)_{\sigma^{*}}$. To get a local parametrization at points that do not belong to this set we simply replace some (or all) of the curves $\beta_{j}, j>g+1$, by their Dehn twists around the corresponding $\alpha_{j}$. In this way we get a local parametrization for $T(\Sigma)$ everywhere. 


\section{References}

[TT] FAthi, A., et al.: Travaux de Thurston sur les surfaces. - Astérisque 66-67, Soc. Math. France, Paris, 1979.

[L] Lehto, O.: Univalent functions and Teichmüller spaces. - To appear.

[MS] SeppäLÄ, M.: Teichmüller spaces of Klein surfaces. - Ann. Acad. Sci. Fenn. Ser. A I Math. Diss. 15, 1978, 1-37.

[SS] SeppäLÄ, M., and T. SoRvali: Parametrization of Möbius groups in a disk. - To appear.

[TS 1] SoRvali, T.: The boundary mapping induced by an isomorphism of covering groups. - Ann. Acad. Sci. Fenn. Ser. A I Math. 526, 1972, 1-31.

[TS 2] SoRvali, T.: Parametrization of free Möbius groups. - Ann. Acad. Sci. Fenn. Ser. A I Math. 579, 1974, 1-11.

[TS 3] Sorvali, T.: On Teichmüller spaces of tori. - Ann. Acad. Sci. Fenn. Ser. A I Math. 1, 1975, 7-11.

University of Helsinki

Department of Mathematics

Hallituskatu 15

SF-00100 Helsinki

Finland

Received 2 April 1984
University of Joensuu

Department of Mathematics

P.O. Box 111

SF-80101 Joensuu

Finland 